\title{
Shaft Inflation and the Planck satellite observations
}

\section{Konstantinos Dimopoulos*}

Consortium for Fundamental Physics, Physics Department, Lancaster University, Lancaster LA1 4YB, UK

E-mail: k.dimopoulosielancaster.ac.uk

\begin{abstract}
A new family of inflation models is introduced and studied. The models are characterised by a scalar potential which, far from the origin, approaches an inflationary plateau in a power-law manner, while near the origin becomes monomial, as in chaotic inflation. The models are obtained in the context of global supersymmetry starting with a superpotential, which interpolates from a generalised monomial to an O'Raifearteagh form for small to large values of the inflaton field respectively. It is demonstrated that the observables obtained, such as the scalar spectral index, its running and the tensor to scalar ratio, are in excellent agreement with the latest observations, without any fine-tuning. Moreover, by widening mildly the shaft in field space, it is shown that sizable tensors can be generated, which may well be observable in the near future.
\end{abstract}

18th International Conference From the Planck Scale to the Electroweak Scale 25-29 May 2015

Ioannina, Greece

* Speaker. 


\section{Introduction}

The latest observations from the Planck satellite $[1,2,3]$ confirm the vanilla predictions of cosmic inflation for the primordial curvature perturbation in that the latter is predominantly Gaussian (non-Gaussianities have not been observed, with upper bound $f_{\mathrm{NL}}^{\text {local }}=0.9 \pm 5.7$ ), adiabatic (no isocurvature contribution has been observed, with upper bound to less that 3\%), statistically isotropic (no statistical anisotropy has been observed, with upper bound to less than $2 \%$ ) and almost scale-invariant, but with a significant red tilt $\left(n_{s}=0.968 \pm 0.006\right)$. Moreover, the Planck data favour canonically normalised, single-field, slow-roll inflation [1]. In fact, in conjunction with other data, Planck seems to favour an inflationary plateau [4].

There have been many examples of such inflationary modes, such as the original $R^{2}$-inflation [5], Higgs inflation [6] or T-model inflation [7]. However, most of these attempts consider an exponential approach to the inflationary plateau. Here we design a model, which approaches the inflationary plateau in a power-law manner, offering distinct observational signatures.

\section{Bottom-up versus top-down approach}

In inflationary model-building one can identify two broad strategies. The top-down scenario corresponds to designing models based on "realistic" constructions, for example inspired by string theory, supergravity etc. Then, one looks for specific signatures in the data (e.g. non-Gaussianity). Since the latest Planck data favour single-field, slow-roll inflation, they seem to support such relatively straightforward constructions.

In contrast, the bottom-up scenario amounts to inflationary model constructions, which are "suggested" by the data, i.e. they are data-inspire "guess-stimates". As such, this approach uses the Early Universe as laboratory to investigate fundamental physics, in the best tradition of particle cosmology. We adopt this strategy (see also Ref. [8]). Our model proposes a power-law approach to the inflationary plateau in the context of global supersymmetry.

\section{The scalar potential for Shaft Inflation}

Consider a toy-model superpotential of the form: $W=M^{2} \Phi^{n q+1} /\left(\Phi^{n}+m^{n}\right)^{q}$, where $n, q$ are real numbers and $M, m$ are mass-scales. For $|\Phi| \gg m$, this superpotential approaches an O' Raifearteagh form $W \simeq M^{2} \Phi$ leading to de Sitter inflation. For $|\Phi| \ll m$, the superpotential becomes $W \propto \Phi^{n q+1}$ leading to chaotic inflation. To simplify it even further, we choose to eliminate the numerator, and take $q=-1 / n$. We end up with the superpotential for Shaft Inflation [8]:

$$
W=M^{2}\left(\Phi^{n}+m^{n}\right)^{1 / n} .
$$

To obtain the scalar potential, we consider $\Phi=\phi e^{i \theta}$, where $\phi, \theta$ are real scalar fields with $\phi>0 .{ }^{1}$ Then the scalar potential is:

$$
V=M^{4}|\Phi|^{2(n-1)}\left|\Phi^{n}+m^{n}\right|^{2\left(\frac{1}{n}-1\right)}=\frac{M^{4} \phi^{2(n-1)}}{\left[\phi^{2 n}+m^{2 n}+2 \cos (n \theta) m^{n} \phi^{n}\right]^{\frac{n-1}{n}}} .
$$

\footnotetext{
${ }^{1}$ A normalisation factor of $1 / \sqrt{2}$ has been absorbed in the mass scales.
} 
The potential is minimised when $n \theta=2 \ell \pi$, with $\ell$ being an integer. Further, noting that $-\phi=\phi e^{i \pi}$, we can make the potential symmetric over the origin $[V(\phi)=V(-\phi)]$ if $n=2 \ell$, i.e. even. In this case,

$$
V(\phi)=M^{4} \phi^{2 n-2}\left(\phi^{n}+m^{n}\right)^{\frac{2}{n}-2},
$$

for all real values of $\phi$. From the above we see that the scalar potential has the desired behaviour, for $n \geq 2$, i.e. it approaches a constant $V \approx M^{4}$ for $\phi \gg m$, while for $\phi \ll m$ the potential becomes monomial, with $V \propto \phi^{2(n-1)}$, see Fig. 1 .

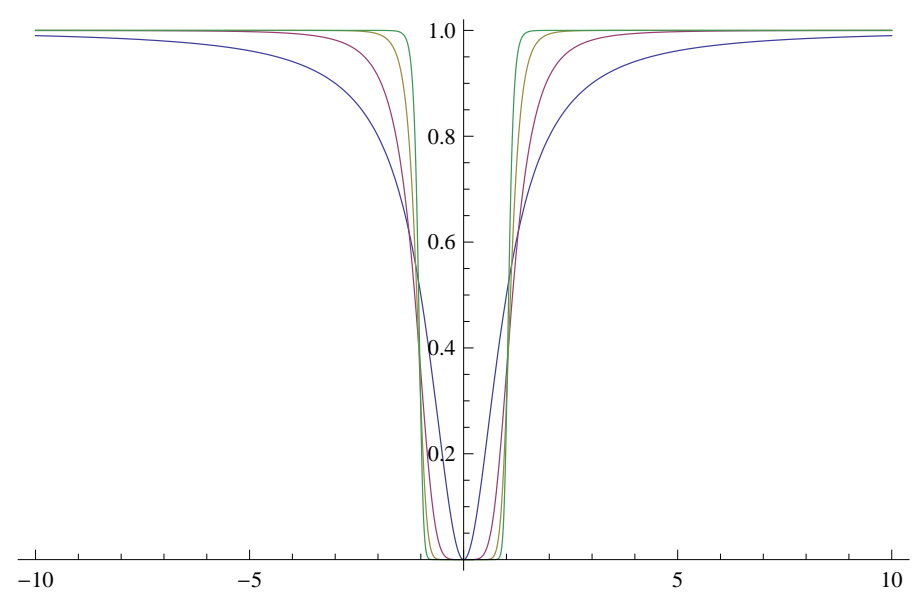

Figure 1: The scalar potential in shaft inflation for $n=2,4,8$ and 16. The shaft becomes sharper as $n$ grows. Far from the origin the potential approximates the inflationary plateau with $V \approx M^{4}$. Near the origin the potential becomes monomial, as in chaotic inflation.

\section{The spectral index and the tensor to scalar ratio}

\subsection{Slow-roll parameters, $n_{s}$ and $r$}

From Eq. (3.3), we readily obtain the slow-roll parameters as

$$
\begin{aligned}
& \varepsilon \equiv \frac{1}{2} m_{P}^{2}\left(\frac{V^{\prime}}{V}\right)^{2}=2(n-1)^{2}\left(\frac{m_{P}}{\phi}\right)^{2}\left(\frac{m^{n}}{\phi^{n}+m^{n}}\right)^{2} \\
& \eta \equiv m_{P}^{2} \frac{V^{\prime \prime}}{V}=2(n-1)\left(\frac{m_{P}}{\phi}\right)^{2}\left(\frac{m^{n}}{\phi^{n}+m^{n}}\right) \frac{(2 n-3) m^{n}-(n+1) \phi^{n}}{\phi^{n}+m^{m}}
\end{aligned}
$$

where the prime denotes derivative with respect to the inflaton field and $m_{P}=2.4 \times 10^{18} \mathrm{GeV}$ is the reduced Planck mass. Hence, the spectral index of the curvature perturbation is

$$
n_{s}=1+2 \eta-6 \varepsilon=1-4(n-1)\left(\frac{m_{P}}{\phi}\right)^{2} \frac{m^{n}\left[(n+1) \phi^{n}+n m^{n}\right]}{\left(\phi^{n}+m^{n}\right)^{2}} .
$$

To rewrite the above as functions of the remaining e-folds of inflation $N$ we have to investigate the end of inflation. It is straightforward to see that inflation is terminated when $|\eta| \simeq 1$ so that, for the end of inflation, we find

$$
\phi_{\mathrm{end}} \simeq m_{P}\left[2\left(n^{2}-1\right) \alpha^{n}\right]^{1 /(n+2)},
$$


where we assumed that $\phi>m$ (so that the potential deviates from a chaotic monomial) and we defined

$$
\alpha \equiv \frac{m}{m_{P}}
$$

Using this, we obtain $\phi(N)$

$$
\begin{aligned}
N=\frac{1}{m_{P}^{2}} \int_{\phi_{\text {end }}}^{\phi} \frac{V}{V^{\prime}} \mathrm{d} \phi & \simeq \frac{1}{2(n-1)(n+2) \alpha^{n}}\left[\left(\frac{\phi}{m_{P}}\right)^{n+2}-\left(\frac{\phi_{\text {end }}}{m_{P}}\right)^{n+2}\right] \\
& \Rightarrow \phi(N) \simeq m_{P}\left[2(n-1)(n+2) \alpha^{n}\left(N+\frac{n+1}{n+2}\right)\right]^{1 /(n+2)} .
\end{aligned}
$$

Inserting the above into Eqs. (4.1) and (4.3) respectively we obtain the tensor to scalar ratio $r$ and the spectral index $n_{s}$ as functions of $N$ :

$$
\begin{aligned}
& r=16 \varepsilon=32(n-1)^{2} \alpha^{\frac{2 n}{n+2}}\left[2(n-1)(n+2)\left(N+\frac{n+1}{n+2}\right)\right]^{-2\left(\frac{n+1}{n+2}\right)} \\
& n_{s}=1-2 \frac{n+1}{n+2}\left(N+\frac{n+1}{n+2}\right)^{-1}
\end{aligned}
$$

Notice that only $r$ is dependent on $m$ (through $\alpha$ ), which means that $r$ can be affected by changing $m$ without disturbing $n_{s}$. We will return to this possibility later.

\subsection{Examples}

To investigate the performance of the model, we consider the two extreme cases for the values of $n$, namely $n=2$ and $n \gg 1$. For illustrative purposes we take $\alpha=1$, i.e. $m=m_{P}$.

\subsection{1 $n=2$}

In this case the scalar potential becomes

$$
V(\phi)=M^{4} \frac{\phi^{2}}{\phi^{2}+m^{2}}
$$

We see that the above can be thought of as a modification of quadratic chaotic inflation, because after the end of inflation, the inflaton field oscillates in a quadratic potential. However, for large values of the inflaton the potential approaches a constant. This potential has been obtained also in S-dual superstring inflation [9] with $\alpha=1 / 4$ and also in radion assisted gauge inflation [10] with $\alpha \sim 10^{-3 / 2}$. In this case, Eqs. (4.8) and (4.9) become

$$
r=\frac{32 \alpha}{\left[8\left(N+\frac{3}{4}\right)\right]^{3 / 2}} \quad \text { and } \quad n_{s}=1-\frac{3}{2}\left(N+\frac{3}{4}\right)^{-1}
$$

From the above, we find the values for $n_{s}$ and $r$, as shown in Table 1. 


\begin{tabular}{|c|c|c|}
\hline$N$ & $n_{s}$ & $r$ \\
\hline \hline 50 & 0.970 & 0.0039 \\
60 & 0.975 & 0.0030 \\
\hline
\end{tabular}

Table 1: Values of $n_{s}(N)$ and $r$ in the case $n=2$.

\subsection{2 $n \gg 1$}

In the opposite extreme $n \gg 1$, Eqs. (4.8) and (4.9) become

$$
r=\frac{8 \alpha^{2}}{n^{2}(N+1)^{2}} \rightarrow 0 \quad \text { and } \quad n_{s}=1-\frac{2}{N+1}
$$

The spectral index is now the same as in the original $R^{2}$ inflation model [5] (also in Higgs inflation [6]), which is not surprising since we expect power-law behaviour to approach the exponential when $n \rightarrow \infty$. The values of $n_{s}$, in this case, are shown in Table 2 .

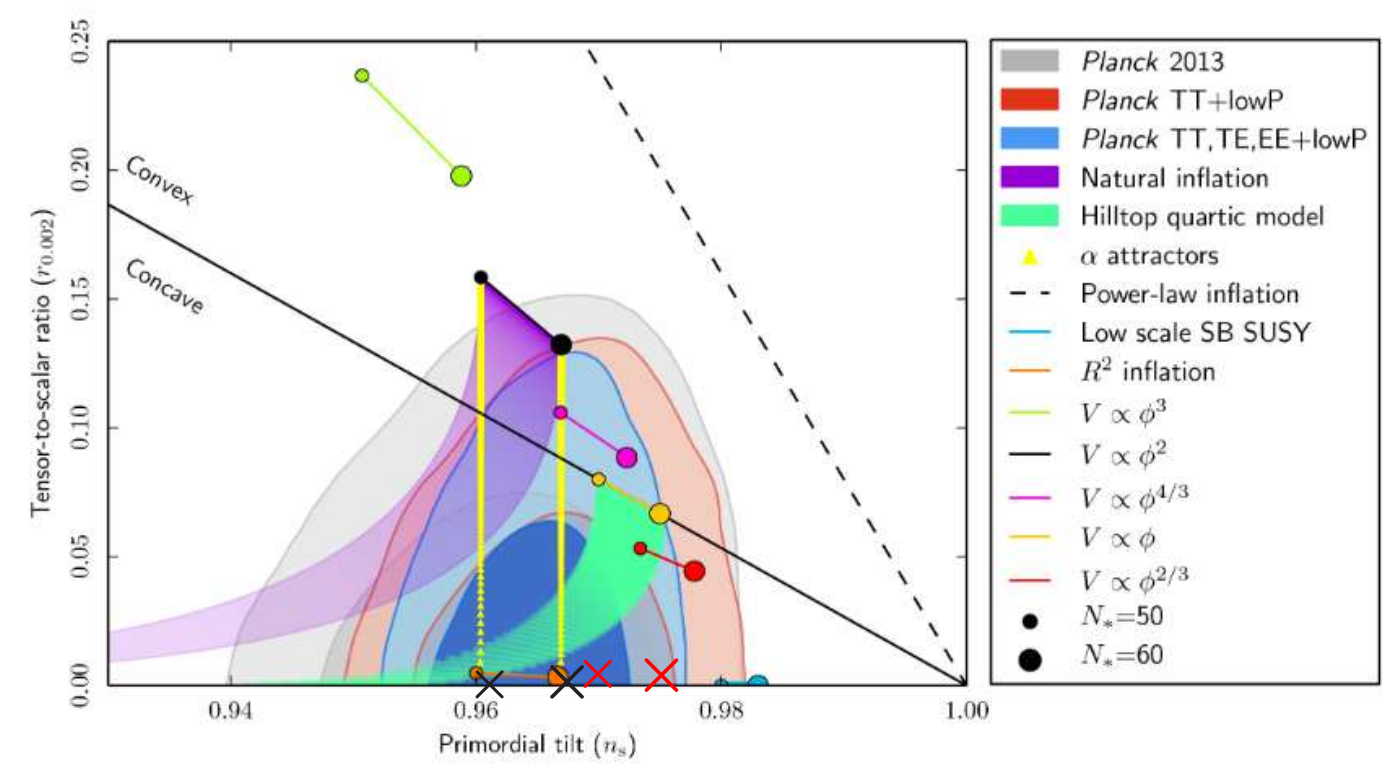

Fig. 12. Marginalized joint $68 \%$ and $95 \%$ CL regions for $n_{\mathrm{s}}$ and $r_{0.002}$ from Planck in combination with other data sets, compared to the theoretical predictions of selected inflationary models.

Figure 2: Shaft inflation for $n=2$ is depicted with the large $\{$ small $\}$ red cross for $N \simeq 60\{N \simeq 50\}$. Shaft inflation for $n \gg 1$ is depicted with the large $\{$ small $\}$ black cross for $N \simeq 60\{N \simeq 50\}$. Intermediate values of $n$ lie in-between the depicted points. As evident, there is excellent agreement with the Planck observations.

From the above, we find that the values for $n_{s}$ and $r$ are very close to the best fit point for the Planck data for all values of $n$, as shown in Fig. $2 .^{2}$

\footnotetext{
${ }^{2}$ The crosses are superimposed to the original Planck paper image, taken from Ref. [1], which includes also the original caption.
} 


\begin{tabular}{|c|c|}
\hline$N$ & $n_{s}$ \\
\hline \hline 50 & 0.961 \\
60 & 0.967 \\
\hline
\end{tabular}

Table 2: Values of $n_{s}(N)$ in the case $n \gg 1($ where $r \approx 0)$.

\begin{tabular}{|c||c|c|c|c|}
\hline$n$ & $n_{s}$ & $r(\alpha=1)$ & $r(\alpha=2 \sqrt{8 \pi} \approx 10)$ & $r(\alpha=5 \sqrt{8 \pi} \approx 25)$ \\
\hline \hline 2 & 0.975 & 0.0030 & 0.0299 & 0.0747 \\
4 & 0.973 & 0.0008 & 0.0168 & 0.0570 \\
6 & 0.971 & 0.0003 & 0.0089 & 0.0352 \\
8 & 0.970 & 0.0001 & 0.0052 & 0.0227 \\
\hline
\end{tabular}

Table 3: Values of $n_{s}$ and $r$ for $N=60$ and $n=2,4,6,8$. Three choices of $\alpha=m / m_{P}$ are depicted, which correspond to $m=m_{P}, m=2 M_{P}$ and $m=5 M_{P}$, where $M_{P}=\sqrt{8 \pi} m_{P}$. It is shown that, with $m$ mildly super-Planckian, $r$ can approach the observational bound $r<0.1$ without affecting $n_{s}$.

\section{Gravitational waves}

Planck observations, in conjunction with BICEP2 and Keck Array data suggest $r \leq 0.1$ [11]. As we have seen, in Shaft Inflation, $r \propto \alpha^{2 n /(n+2)}$, while there is no $\alpha$-dependence of $n_{s}$. Thus, by changing $m, r$ can vary without affecting the spectral index (c.f. Eq. (4.5)). Therefore, sizeable tensors can be attained by widening the shaft in field space. Indeed, rendering $m$ mildly superPlanckian can produce potentially observable values of $r$ as shown in Table 3, where Eq. (4.5) suggests that $m=\alpha m_{P}=\frac{\alpha}{\sqrt{8 \pi}} M_{P}$, with $M_{P}=1.2 \times 10^{19} \mathrm{GeV}$ being the Planck mass. Thus, we see that with $m \simeq 5 M_{P}$ we can have $r \simeq 0.07$, which is on the verge of observability. This is shown clearly in Fig, 3.

\section{More on Shaft Inflation}

The running of the spectral index is easily obtained as

$$
\frac{\mathrm{d} n_{s}}{\mathrm{~d} \ln k}=-\frac{2\left(\frac{n+1}{n+2}\right)}{\left(N+\frac{n+1}{n+2}\right)^{2}} .
$$

In the two extreme cases, this gives

$$
\begin{aligned}
& n=2: \frac{\mathrm{d} n_{s}}{\mathrm{~d} \ln k}=-\frac{3}{2\left(N+\frac{3}{4}\right)^{2}}=-4.064 \times 10^{-4} \\
& n \gg 1: \frac{\mathrm{d} n_{s}}{\mathrm{~d} \ln k}=-\frac{2}{(N+1)^{2}}=-5.375 \times 10^{-4}
\end{aligned}
$$

where the numerical values correspond to $N=60$. Thus, for all values of $n$, we the above suggests: $\frac{\mathrm{d} n_{s}}{\mathrm{~d} \ln k} \approx-(4-5) \times 10^{-4}$, which is in agreement with the Planck findings: $\frac{\mathrm{d} n_{s}}{\mathrm{~d} \ln k}=-0.003 \pm 0.007$. 


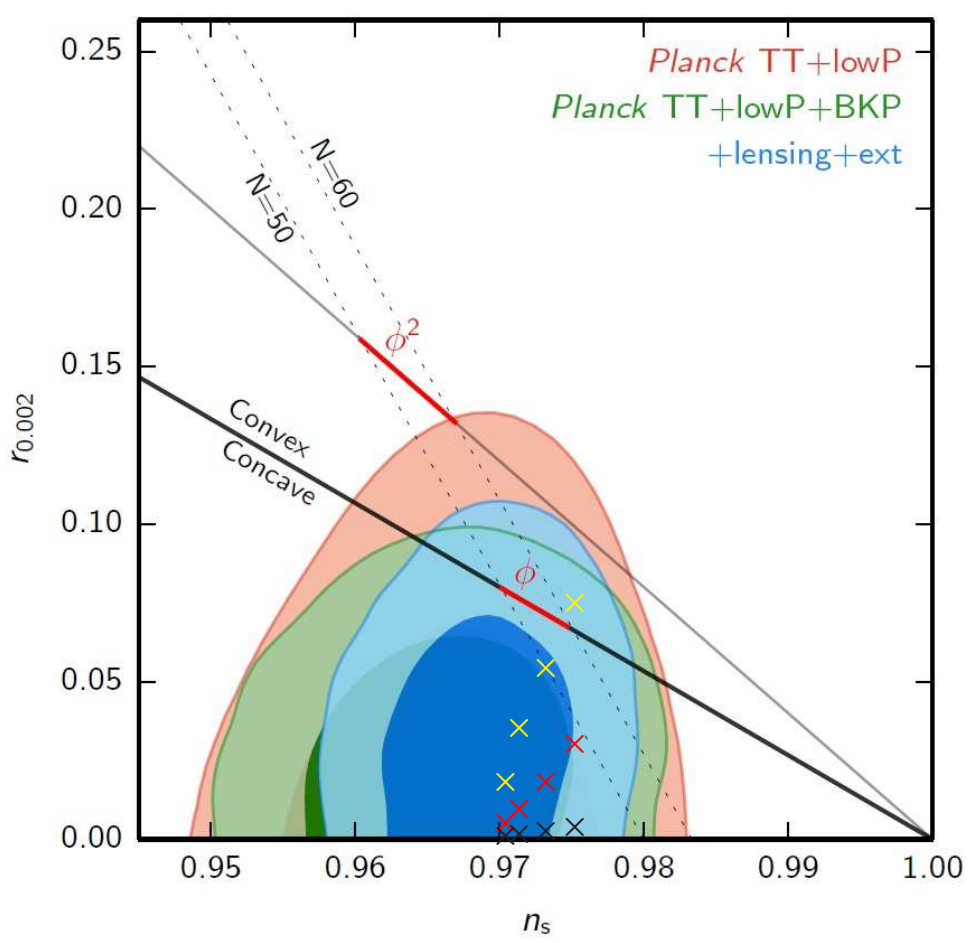

Figure 3: Shaft inflation predictions for $N=60$. The crosses in the image correspond to $n=2,4,6,8$ as depicted from right to left. Black crosses correspond to $\alpha=1\left(m=m_{P}\right)$, red crosses correspond to $\alpha=2 \sqrt{8 \pi} \approx 10\left(m=2 M_{P}\right)$ and yellow crosses correspond to $\alpha=5 \sqrt{8 \pi} \approx 25\left(m=5 M_{P}\right)$. It is evident that, for mildly super-Planckian values of $m$ the model predictions lie at the verge of observability.

Finally, the inflationary scale is determined by the COBE constraint

$$
\sqrt{\mathscr{P}_{\zeta}}=\frac{1}{2 \sqrt{3} \pi} \frac{V^{3 / 2}}{m_{P}^{3}\left|V^{\prime}\right|}
$$

where $\mathscr{P}_{\zeta}=(2.208 \pm 0.075) \times 10^{-9}$ is the spectrum of the curvature perturbation [2]. This provides an estimate for the required value of $M$

$$
\left(\frac{M}{m_{P}}\right)^{2}=4 \sqrt{3}(n-1) \alpha^{-\frac{n}{n+2}} \pi \sqrt{\mathscr{P}_{\zeta}}\left[2(n-1)(n+2)\left(N+\frac{n+1}{n+2}\right)\right]^{-\frac{n+1}{n+2}}
$$

For illustrative purposes, using $\sqrt{\mathscr{P}_{\zeta}} \simeq 4.7 \times 10^{-5}, n=2, \alpha=1$ (i.e. $m=m_{P}$ ) and $N=60$ we find $M=7.7 \times 10^{15} \mathrm{GeV}$, which is very near the scale of grand unification, as expected.

\section{Conclusions}

Planck data favour single-field, slow-roll inflation, characterised by a scalar potential which approaches an inflationary plateau. In contrast to many other successful models, Shaft Inflation approaches this plateau in a power-law manner. Shaft Inflation is based on a simple superpotential: $W=M^{2}\left(\Phi^{n}+m^{n}\right)^{1 / n}$. Without any fine tuning $\left(m \sim m_{P}\right.$ and $M \sim 10^{16} \mathrm{GeV}$, i.e. the scale of grand unification) Shaft Inflation produces a scalar spectral index very close to the Planck sweet spot with very small (negative) running, in agreement with Planck. Rendering $m$ mildly super-Planckian one 
can easily obtain potentially observable tensors without affecting the spectral index. The challenge in now to obtain realistic setups which can realise the (deceptively) simple Shaft Inflation superpotential.

\section{Acknowledgements}

$\mathrm{KD}$ is supported (in part) by the Lancaster-Manchester-Sheffield Consortium for Fundamental Physics under STFC grant ST/L000520/1.

\section{References}

[1] P. A. R. Ade et al. [Planck Collaboration], arXiv:1502.02114 [astro-ph.CO].

[2] P. A. R. Ade et al. [Planck Collaboration], Planck 2015 results. XIII. Cosmological parameters, arXiv:1502.01589 [astro-ph.CO];

[3] P. A. R. Ade et al. [Planck Collaboration], Planck 2015 results. XVII. Constraints on primordial non-Gaussianity, arXiv:1502.01592 [astro-ph.CO].

[4] J. Martin, C. Ringeval, R. Trotta and V. Vennin, The Best Inflationary Models After Planck, JCAP 1403 (2014) 039 [arXiv:1312.3529 [astro-ph.CO]].

[5] A. A. Starobinsky, A New Type of Isotropic Cosmological Models Without Singularity, Phys. Lett. B 91 (1980) 99; Sov. Astron. Lett. 9 (1983) 302.

[6] F. L. Bezrukov and M. Shaposhnikov, The Standard Model Higgs boson as the inflaton, Phys. Lett. B 659 (2008) 703 [arXiv:0710.3755 [hep-th]].

[7] A. Linde, Inflationary Cosmology after Planck 2013, arXiv:1402.0526 [hep-th].

[8] K. Dimopoulos, Phys. Lett. B 735 (2014) 75 [arXiv:1403.4071 [hep-ph]].

[9] A. de la Macorra and S. Lola, Inflation in S dual superstring models, Phys. Lett. B 373 (1996) 299 [hep-ph/9511470].

[10] M. Fairbairn, L. Lopez Honorez and M. H. G. Tytgat, Radion assisted gauge inflation, Phys. Rev. D 67 (2003) 101302 [hep-ph/0302160].

[11] P. A. R. Ade et al. [BICEP2 and Planck Collaborations], Joint Analysis of BICEP2/KeckArray and Planck Data, Phys. Rev. Lett. 114 (2015) 101301 [arXiv:1502.00612 [astro-ph.CO]]. 